\title{
Understanding Users Intent by Deducing Domain Knowledge Hidden in Web Search Query Keywords
}

\author{
Nachiket B. Kamat \\ Computer Engineering Department \\ Goa College of Engineering \\ Farmagudi-Ponda, Goa, India
}

\author{
Geeta Varkey \\ Computer Engineering Department \\ Goa College of Engineering \\ Farmagudi-Ponda, Goa, India
}

\begin{abstract}
Search Engines are used by people on a daily basis to retrieve information from the web. When an ambiguous word is present in a query, specific sense of the keyword is not considered during the search process. Search engines return a large amount of web pages as results from all the possible contexts. Users tend to browse only few pages. Improving quality of retrieved results is a challenge and needs research. There is no attempt made by search engines to establish the context/domain/intent of the query keyword, before forwarding query to the engine. Current research trends in word sense disambiguation and availability of huge knowledge bases like BabelNet which encompasses WordNet and Wikipedia, together with WordNet Domains in which senses are annotated with domain labels indicates scope for developing methods capable of performing semantic analysis of search engine queries. In this paper, an algorithm for automatically establishing context in web search queries using modern automatic word sense disambiguation technique is proposed. Further, it is illustrated how Query Expansion can be performed using the interactive feedback and deduced domain of the query keyword. The domain knowledge and query expansion helps retrieve high quality results, specific to the user's intent.
\end{abstract}

\section{General Terms}

Information Retrieval, Search Engine, Natural Language Processing

\section{Keywords}

Query Expansion, Word Sense Disambiguation, Context Establishment

\section{INTRODUCTION}

Search Engines are used by people on a daily basis to retrieve information from the web. Amongst the thousands of results retrieved by the search engine, users tend to browse only few pages. When an ambiguous word is present in a query, multiple meanings of that word are not considered. Thus, search engines multiple web pages as results, from all the possible contexts. Hence, improving quality of retrieved results is a challenge and needs research.

Human language is inherently ambiguous. In English, many words can be interpreted in different ways, depending upon the words which occur along with it in the query. For instance, the English word 'bank' can mean a sloping piece of land near river body or a financial institution. For humans, identifying the correct sense is a trivial task due to presence of huge world knowledge for past many years in English language. However, this is a difficult task for a computer. If search engine is able to automatically identify the correct sense of word in the query context, then it can use this information to establish the domain of query keywords and thus build a better query with context information before the search process starts.

Word Sense Disambiguation is the technique to identify the correct sense of a particular word in a given context. Word sense disambiguation (WSD) has been described as an AIcomplete problem, a problem whose difficulty is equivalent to solving central problems in artificial intelligence (AI). Current research suggests the availability of state-of-art algorithms and research resources that enable researchers to pursue study in application of state-of-art WSD in semantic search \& natural language processing and hence, in better Information retrieval. Query Expansion (QE) is the building of a new query from an old one. $\mathrm{QE}$ techniques include: Use of thesaurus, query reformulations based on query log mining and relevance feedback. However, none of the publications discuss query expansion using the domain knowledge hidden behind keywords in the query. The word 'context' in this paper refers to the domain/category of the keyword in query, unless otherwise specified.

In this paper, an algorithm for establishing context of keywords in web query using state-of-art automatic word sense disambiguation algorithm, interactive feedback and WordNet Domains is proposed. Further, it is illustrated how Query Expansion can be performed using the identified domain, which can help in retrieving better search results in terms of intended domain of query keywords.

The paper is organized as follows. Related work on word sense disambiguation and query expansion with the past and the present works is briefly discussed in Section 2. In Section 3 , the motivational factors for taking up this work are given, followed by the proposed algorithm in Section 4. Section 5 provides experimental results. Section 6 concludes the paper along with directions for future research followed by the acknowledgement and references in Section 7 and 8 respectively.

\section{Related Work}

The task of WSD [7] is a historical one in the field of Natural Language Processing (NLP) which was earlier attacked using AI techniques. However, later the release of large-scale lexical resources enabled automatic methods for knowledge extraction. Lesk 1986 [8] presented a method of word sense disambiguation that compared the definitions/gloss of the ambiguous word to the definitions/gloss of the neighboring words. Sense whose overlap is largest is selected as the target sense. Unfortunately; Lesk's approach is very sensitive to the exact wording of definitions and dictionary glosses are short. Later, Banerjee and Pederson in [9] came up with an adapted 
Lesk algorithm in which they introduced a window of context containing of a few words to the right \& left of the target word and only applied the gloss overlaps method to words within this window of context.

Patwardhan, Banerjee \& Pederson studied measures of semantic relatedness and generalized the adapted Lesk algorithm by [9] to a method of WSD based on semantic relatedness measures [10]. Subsequently, authors have provided implementations in Perl of research resources called WordNet::Similarity, for computation of semantic relatedness from WordNet in [10] and for WSD called SenseRelate::TargetWord in [11] and SenseRelate::AllWords in [11]. [12]introduces a measure of extended gloss overlap to compute semantic relatedness, which expands the glosses of the words being compared to include glosses of concepts that are known to be related through explicit relations (e.g.: partof, has-a, is-a, kind-of, etc.) in the lexical resource like WordNet[19]. [13] applied the same techniques to an allwords disambiguation task using an algorithm that computes a score for each sense of the target word.

Navigli in [1] suggests the use of Wikipedia along with WordNet. Author claims that in recent years progress has been made that has led to a considerable boost in disambiguation performance, from about $65 \%$ to $82-83 \%$ accuracy. In [2], authors have come up with a methodology to automatically extend WordNet with large amounts of semantic relations from an encyclopedic resource, namely Wikipedia to form WordNet. In [14] the authors discuss further improvement to the above paper [2] to create BabelNet, a very large multilingual knowledge base. The existing WordNet++ is enriched with lexical information for multiple languages. This fully-automated approach produces a large-scale lexical resource with high accuracy.

Multilingual joint approach [15] involves exploiting of different languages together at the same time. The method exploits BabelNet, a very large multilingual knowledge base, to perform graph-based WSD across different languages. State-of-art research shows that the recent developments in lexical knowledge-base resources like WordNet++ \& BabelNet makes knowledge based WSD a competitor and in some cases also outperforms other supervised \& unsupervised methods.

[17] suggests a controversy over whether WSD really benefits IR. In the case where the answer to this question is no, WSD did not help due to the low accuracy achieved by automatic WSD, but that improvement was possible if WSD could be done with a high enough accuracy. Current research trends in word sense disambiguation[16] and availability of huge knowledge bases like BabelNet which encompasses WordNet and Wikipedia, together with WordNet Domains in which senses are annotated with domain labels seems to indicate that the time is ripe for developing methods capable of performing semantic analysis of search engine queries.

Query Expansion (QE) techniques include[4]: Use of thesaurus, query reformulations based on query $\log$ mining and relevance feedback. Query logs [6] only show the recorded actions and not the intent behind the queries. For example, query logs cannot tell whether a user did not click because the information they were looking for was found on the results page, or because the results were unsatisfying. However, none of the publications discuss query expansion by using context/domain of the keyword.
WordNet Domains (WNDomains) [18] is a lexical resource created in a semi-automatic way by augmenting WordNet with domain labels. WordNet Synsets have been annotated with at least one semantic domain label, selected from a set of about two hundred labels structured according the WordNet Domain Hierarchy. The resource is in form of a database which contains mapping between the WordNet 2.0 synsetID and the domain for that synset.

\section{Motivational Factors}

The best search engines like Google provide high quality of results by involving users in the search process using auto complete, related searches and did you mean features. However, there is no attempt made at establishing the context/domain of keywords in the query. The domain of keyword can enable the search engine to aid user in developing better query by performing query expansion before fetching the $1^{\text {st }}$ set of results.

Word Sense Disambiguation is the technique to identify the correct sense of keywords in a query. The major motivation was the literature survey of recent research in word sense disambiguation techniques and knowledge-base resources [16]. As mentioned in [1], much work is still needed to prove that a proper injection of semantics into real-world applications like semantic search is always beneficial. Latest research suggests that knowledge based WSD techniques outperform other supervised \& un-supervised techniques [2]. Besides, knowledge-rich WSD has been shown to achieve high performance across domains and to compete with supervised methods on a variety of lexical disambiguation tasks [3]. Recent research has introduced BabelNet[14] which complements existing resources like WordNet with millions of more semantic relations harvested from Wikipedia and mapped into WordNet via an accurate mapping algorithm with experimentally proven results. If word sense disambiguation is to be of any use to an IR system, the disambiguator must be able to resolve word senses to a high degree of accuracy [17]. The state-of-art WSD systems provide better accuracy levels and hence their application to IR needs research.

The state-of-art word sense disambiguation algorithms have a much higher accuracy then other supervised/unsupervised algorithms. However, since WSD is still an open research problem and most web queries are short, to ensure that the system captures the intended user's intent accurately, interactivity is provided by allowing user to choose the correct sense of each query keyword. The WSD algorithm aids the user by suggesting the right sense, but eventually choice is in hands of the user. The involvement of user in initial phase of sense selection helps to provide a more accurate context establishment, thus better quality of results. [20] gives a technique that helps in offering more accurate results, especially in case of Homographs. Homograph is a word that shares the same written form but has different meanings. They show how senses of words can play an important role in offering accurate search results. 


\section{Proposed Methodology}

\section{Fig 1: System architecture}

Algorithm:-

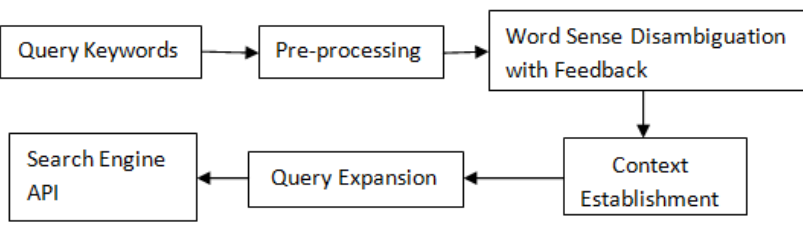

1. Retrieve the users query which will be string of tokens

2. Apply pre-processing

a. Tokenize

b. POS tagging (nouns/verbs)

c. Stop-Words removal

3. For each token (sense tag based on context)

a. Identify all the senses of that word within BabelNet

b. Look for their connecting paths

c. Merge the identified paths within a single graph

d. Score the graph nodes using OutDegree measure

e. Suggest to the user the node with highest score as the identified sense of keyword

f. Allow user to make choice by providing user with list of word senses

g. If a choice is made, then capture the synsetID of user's choice

4. To establish context: - Map the synsetID of the chosen term with the WordNet Domains database consisting of WordNet synsets annotated with domain labels.

5. Append the domain/context along with the keyword

6. Forward the re-formulated query to the search engine via API interface.

\section{Experimental Results}

\section{A. Experimental Setup}

Experiments were carried out on Intel core i3 $2.40 \mathrm{GHz}$ processor with 1.5GB RAM, Ubuntu (Linux) OS. Programs were implemented in Java on NetBeans7.3SDK. Algorithm was applied for the query - "Crane parts".

\section{B. Proposed Algorithm's Output}

The results returned via Google search for the query 'Crane parts' is as shown in fig 2. However, the intent of the search is not taken into account, neither is an attempt made to establish context. The top 10 search results for 'Crane parts' are in terms of the 'machine' sense. The 'bird' sense of word crane is not considered in search which could have been the intent of the user.

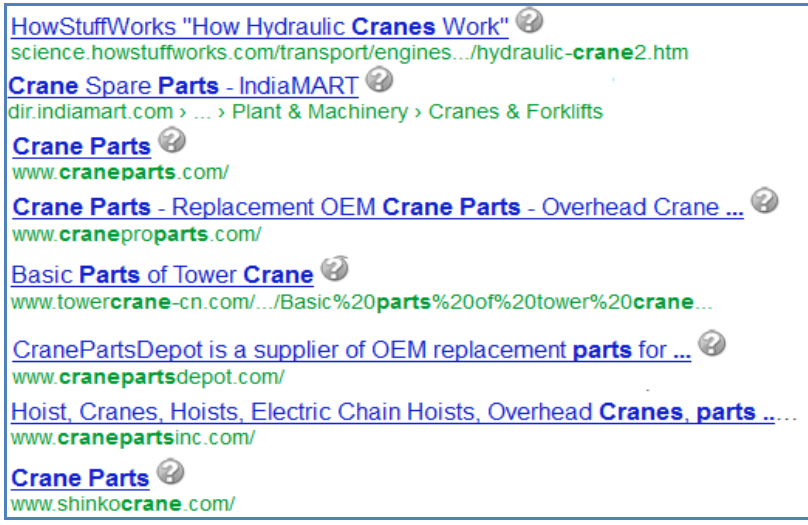

Fig 2: Search results for initial query

The proposed algorithm tries to disambiguate crane with respect to the context by traversing the BabelNet graph and scoring the nodes using the OutDegree measure. The WSD algorithm's output is suggested to the user along with list of other senses for that word. Since the user has chosen the bird sense, results obtained are as shown in fig 3 .

Crane (bird) - Wikipedia, the free encyclopedia
en.wikipedia.org/wiki/Crane_(bird)
Common Crane - Wikipedia, the free encyclopedia
en.wikipedia.org/wiki/Common_Crane
BioKIDS - Kids' Inquiry of Diverse Species, Critter Catalog, Grus ...
www.biokids.umich.edu/critters/Grus_canadensis/
Video -- Whooping Cranes -_ National Geographic
crane - definition and meaning 8
www.wordnik.com/words/crane
There are about 15 closely similar species, found in many
Birds of Minnesota: Sandhill Crane: Minnesota DNR 8
www.dnr.state.mn.us > Nature > Animals > Birds
The Earthlife Web - The Gruidae or Cranes
www.earthlife.net/birds/cranes.html
Siberian Crane - Northern Prairie Wildlife Research Center - USGS
www.npwrc.usgs.gov/resource/birds/cranes/grusleuc.htm

Fig 3: Search results for re-formulated query

The system provides context specific results since the system attempts to understand the query keyword domain and hence the intent which can be seen in the figure above.

\section{Conclusion}

Previously there were not enough resources for performing accurate word sense disambiguation but recently there has been a lot of progress in developing knowledge bases which provide a vast amount of useful and correct semantic relations. Further, it has been experimentally proven that state-of-art knowledge based word sense disambiguation techniques utilizing such huge state-of-art knowledge bases compete \& in some cases they outperform other supervised \& unsupervised techniques. Given the fact that there are techniques like relevance feedback and mining of logs that contribute to re-formulation of query, these techniques highly depend upon the initial results fetched by the search engine/clicked by the user. Users usually do not prefer that their query data is mined and evaluated due to privacy reasons. Query logs only show the recorded actions and not the intent behind the queries because the user may have opened the page to check its contents and evaluate whether the contents of the page are actually relevant to his query. Logs need to be monitored over a long period of time to give good results. Hence, query expansion by establishing context has scope. 
The proposed system has a standard web interface consisting of a single input box that accepts user's query. The query is pre-processed and knowledge based state-of-art Word Sense Disambiguation algorithm is applied to each word to identify its correct sense amongst all the senses of that word. WSD algorithm sense tags each word based on the context (surrounding words) of user's query. The synsetID of the selected sense is used to map into the database of WordNet domains to identify the respective domain category label/context of each word. The domain label is then appended along with the original keyword and this newly formed query is forwarded to the Search Engine via an API interface. Thus the system establishes context and also provides query re-formulation which will eventually give results with respect to the context of the particular sense of word used in the query, that is, specific to the user's intent.

The proposed system provides context specific results since the system attempts to understand the query keyword domain and hence the intent. Thus, retrieved results are relevant to the intended sense of the keyword in users query. Future work in this direction would be to develop a page re-ranking algorithm that will re-rank the set of first $\mathrm{N}$ web pages retrieved by the search engine by utilizing a scoring function that will take into account the bag of words consisting of keywords in the query, as well as the synonyms, hyponym and related words of each keyword along with the context/domain of keywords to be compared against each page in result set. Thus the pages relevant to searchers interest will be displayed with a higher rank. The outcome will be that the newly ranked result set will provide higher rank to pages that are similar to the intent of the user. Further, the domain information can be used to identify the searchers interests over a period of time. The context establishment algorithm can be used for various fields of computer science like information retrieval, social media mining and others as applicable.

\section{Acknowledgment}

This work was performed as part of a Master of Engineering thesis in project titled Context Aware Web Search Engine. We want to acknowledge the contribution of our colleagues from Goa Engineering College for all the support that was provided.

\section{References}

[1] Roberto Navigli, "A Quick Tour of Word Sense Disambiguation, Induction and Related Approaches", M. Bielikov'a et al. (Eds.): SOFSEM 2012, LNCS 7147, pp. 115-129, Springer-Verlag Berlin Heidelberg, 2012

[2] Simone Paolo Ponzetto, Roberto Navigli, "Knowledgerich Word Sense Disambiguation Rivaling Supervised Systems", Proceedings of the 48th Annual Meeting of the Association for Computational Linguistics, pages 15221531, Uppsala, Sweden, July 2010

[3] Roberto Navigli and Simone Paolo Ponzetto, "Multilingual WSD with Just a Few Lines of Code: the BabelNet API", 2012

[4] Yogesh Kakde, "A Survey of Query Expansion until June 2012”, Indian Institute of Technology, Bombay, 2012

[5] Ian Ruthven, "Re-examining the Potential Effectiveness of Interactive Query Expansion", SIGIR'03, Toronto, Canada, 2003
[6] Jeff Huang, Efthimis N. Efthimiadis, "Analyzing and Evaluating Query Reformulation Strategies in Web Search Logs”, CIKM'09, Hong Kong, China, 2009

[7] Roberto Navigli, "Word Sense Disambiguation: A Survey", ACM Computing Surveys, Vol. 41, No. 2, Article 10, February 2009

[8] Michael Lesk, "Automatic Sense Disambiguation Using Machine Readable Dictionaries: How to Tell a Pine Cone from an Ice Cream Cone", ACM, 1986-87

[9] Satanjeev Banerjee, Ted Pederson, “An Adapted Lesk Algorithm for Word Sense Disambiguation Using WordNet", 2002

[10] Patwardhan, Banerjee, Pederson, "Using measures of semantic relatedness for Word Senes Disambiguation", Proceedings of the Fourth International Conference on Intelligent Text Processing and Computational Linguistics, Mexico City, Mexico, February 2003

[11] Ted Pedersen and Varada Kolhatkar, "WordNet::SenseRelate::AllWords -A Broad Coverage Word Sense Tagger that Maximizes Semantic Relatedness"

[12] Satanjeev Banerjee, Ted Pedersen, "Extended Gloss Overlaps as a Measure of Semantic Relatedness"

[13] Jason Michelizzi, "Semantic Relatedness Applied to All Words Sense Disambiguation", Masters thesis, University of Minnesota, July 2005

[14] Roberto Navigli, Simone Paolo Ponzetto, "BabelNet: Building a Very Large Multilingual Semantic Network", Proceedings of the 48th Annual Meeting of the Association for Computational Linguistics, pages 216225, Uppsala, Sweden, July 2010

[15] Roberto Navigli and Simone Paolo Ponzetto, "Joining Forces Pays Off: Multilingual JointWord Sense Disambiguation", Proceedings of the 2012 Joint Conference on Empirical Methods in Natural Language Processing and Computational Natural Language Learning, pages 1399-1410, Jeju Island, Korea, July 2012

[16] Roberto Navigli, Simone Paolo Ponzetto, "BabelNet: The automatic construction, evaluation and application of a wide-coverage multilingual semantic network", Artificial Intelligence 193 (2012) 217-250, August 2012

[17] Hwee Tou Ng, "Does Word Sense Disambiguation Improve Information Retrieval?", ESAIR'11, Glasgow, Scotland, UK, 2011

[18] Luisa Bentivogli, Pamela Forner, Bernardo Magnini and Emanuele Pianta."Revising WordNet Domains Hierarchy: Semantics, Coverage, and Balancing", In Proceedings of COLING 2004 Workshop on "Multilingual Linguistic Resources", Geneva, Switzerland, August 28, 2004, pp. 101-108.

[19] George A. Miller, Richard Beckwith, Christiane Fellbaum, Derek Gross, KatherineMiller, "Introduction to WordNet: An On-line Lexical Database", August 1993

[20] Rekha Jain, G. N. Purohit, "Sense Disambiguation Technique For Providing More Accurate Results in Web Search", International Journal on Web Service Computing (IJWSC), Vol. 3, No. 3, September 2012 